\title{
Determination of fatty acid composition in seed oil of rapeseed (Brassica napus L.) by mutated alleles of the FAD3 desaturase genes
}

\author{
Jan Bocianowski • Katarzyna Mikołajczyk • \\ Iwona Bartkowiak-Broda
}

Received: 16 March 2011 /Revised: 21 July 2011 / Accepted: 30 August 2011 /Published online: 13 September 2011

(C) The Author(s) 2011. This article is published with open access at Springerlink.com

\begin{abstract}
One of the goals in oilseed rape programs is to develop genotypes producing oil with low linolenic acid content $(\mathrm{C} 18: 3, \leq 3 \%)$. Low linolenic mutant lines of canola rapeseed were obtained via chemical mutagenesis at the Plant Breeding and Acclimatization Institute - NRI, in Poznan, Poland, and allele-specific SNP markers were designed for monitoring of two statistically important single nucleotide polymorphisms detected by SNaPshot analysis in two FAD3 desaturase genes, BnaA.FAD3 and BnaC.FAD3, respectively. Strong negative correlation between the presence of mutant alleles of the genes and linolenic acid content was revealed by analysis of variance. In this paper we present detailed characteristics of the markers by estimation of the additive and dominance effects of the $F A D 3$ genes with respect to particular fatty acid content in seed oil, as well as by calculation of the phenotypic variation of seed oil fatty acid composition accounted by particular allele-specific marker. The obtained percentage of variation in fatty acid composition was considerable only for linolenic acid content and equaled $35.6 \%$ for BnaA. $F A D 3$ and $39.3 \%$ for BnaC.FAD3, whereas the total percentage of variation in linolenic acid content was $53.2 \%$ when accounted for mutations in both genes simultaneously. Our results revealed high specificity of the
\end{abstract}

J. Bocianowski $(\bowtie)$

Department of Mathematical and Statistical Methods,

Poznań University of Life Sciences,

Wojska Polskiego 28,

60-637 Poznan, Poland

e-mail: jboc@up.poznan.pl

K. Mikołajczyk $\cdot$ I. Bartkowiak-Broda

Research Division in Poznan, Plant Breeding and Acclimatization

Institute - National Research Institute,

Strzeszynska 36 ,

60-479 Poznan, Poland markers for effective monitoring of the wild-type and mutated alleles of the Brassica napus FAD3 desaturase genes in the low linolenic mutant recombinants in breeding programs.

Keywords Brassica napus L. FAD3 desaturase · Fatty acid composition · Genetic effects · Seed oil · SNaPshot analysis

\section{Introduction}

Oilseed rape (Brassica napus L.) is the second-most important oil crop of the moderate climate zone. Seeds of the double low varieties (canola, '00', with very low glucosinolates content in seed meal and with no erucic acid in seed oil) produce oil containing, on average: $7 \%$ of saturated fatty acids - palmitic (C16:0) and stearic (C18:0), and polyunsaturated fatty acids - oleic (C18:1, 61\%), linoleic $(\mathrm{C} 18: 2,20 \%)$, linolenic $(\mathrm{C} 18: 3,10 \%)$ and eicosenoic fatty acid (C20:1, 1\%). Due to the food- and non-food use of the oil, rapeseed breeding forms characterized by diversified seed oil fatty acid content are of great value on the oil crop market, and one of the breeding goals is to develop genotypes producing oil with low linolenic acid content $(\mathrm{C} 18: 3, \leq 3 \%)$. Such oil is desirable for deep frying, as low content of polyunsaturated linolenic acid prevents oxidation and rancidation of the oil, which is crucial for healty food production. In addition, oil with low linolenic acid content makes an important source of raw material for biofuel production, due to high stability of such oil. Genetic analyses revealed that in B. napus low linolenic acid content was controlled by the two fatty acid desaturase 3 genes (FAD3), named BnaA.FAD3 and BnaC.FAD3, according to the standardized gene nomenclature for the Brassica genus (Østergaard and King 2008). The genes are located in the $\mathrm{A}$ and $\mathrm{C}$ genomes of $B$. napus 
Table 1 Regression analysis of the mutations in the FAD3 genes on particular fatty acid content in seed oil; C16:0, palmitic, C18:0, stearic, C18:1, oleic, C18:2, linoleic, C18:3, linolenic, C20:1, eicosenoic acid; SNP - genotyping of allelic forms of the FAD3 desaturase genes with the use of the SNaPshot assay, in the BnaA. FAD3 gene (FAD3A) and in the BnaC.FAD3 gene (FAD3C), respectively

\begin{tabular}{llllll}
\hline SNP & Trait & $\begin{array}{l}\text { Estimates of regression } \\
\text { coefficients }\end{array}$ & P-values & $\begin{array}{l}\text { Percentage variation } \\
\text { accounted }\end{array}$ & $\begin{array}{l}\text { Standard error } \\
\text { of observations }\end{array}$ \\
\hline FAD3A & $\mathrm{C} 16: 0$ & 0.1355 & $<0.001$ & 3.0 & 0.550 \\
FAD3C & $\mathrm{C} 16: 0$ & 0.065 & 0.051 & 0.6 & 0.557 \\
FAD3A, FAD3C & $\mathrm{C} 16: 0$ & $0.13,0.02$ & $<0.001,0.662$ & 2.8 & 0.551 \\
FAD3A & $\mathrm{C} 18: 0$ & -0.1548 & $<0.001$ & 5.9 & 0.448 \\
FAD3C & $\mathrm{C} 18: 0$ & -0.1213 & $<0.001$ & 4.1 & 0.452 \\
FAD3A, FAD3C & $\mathrm{C} 18: 0$ & $-0.12,-0.07$ & $<0.001,0.011$ & 7.0 & 0.445 \\
FAD3A & $\mathrm{C} 18: 1$ & -0.587 & 0.027 & 0.9 & 4.140 \\
FAD3C & $\mathrm{C} 18: 1$ & 0.402 & 0.106 & 0.4 & 4.150 \\
FAD3A, FAD3C & $\mathrm{C} 18: 1$ & $-0.92,0.75$ & $0.002,0.005$ & 2.3 & 3.730 \\
FAD3A & $\mathrm{C} 18: 2$ & -0.98 & $<0.001$ & 3.4 & 3.490 \\
FAD3C & $\mathrm{C} 18: 2$ & -1.91 & $<0.001$ & 15.5 & 3.490 \\
FAD3A, FAD3C & $\mathrm{C} 18: 2$ & $-0.17,-1.85$ & $0.490,<0.001$ & 15.4 & 1.580 \\
FAD3A & $\mathrm{C} 18: 3$ & 1.606 & $<0.001$ & 35.6 & 1.540 \\
FAD3C & $\mathrm{C} 18: 3$ & 1.5738 & $<0.001$ & 39.3 & 1.350 \\
FAD3A, FAD3C & $\mathrm{C} 18: 3$ & $1.10,1.15$ & $<0.001,<0.001$ & 53.2 & 0.79 \\
FAD3A & $\mathrm{C} 20: 1$ & -0.0186 & 0.129 & 0.3 & 0.191 \\
FAD3C & $\mathrm{C} 20: 1$ & -0.0128 & 0.264 & 0.1 & 0.191 \\
FAD3A, FAD3C & $\mathrm{C} 20: 1$ & $-0.02,-0.001$ & $0.245,0.588$ & 0.1 & 0.191 \\
FA & & &
\end{tabular}

(AACC amphidiploid genome) (Barret et al. 1999; Jourdren et al. 1996), and were mapped by Hu et al. (2006) on the N4 (A4) and N14 (C4) linkage groups, respectively.

Low linolenic mutant lines of canola rapeseed were obtained via chemical mutagenesis at the Plant Breeding and Acclimatization Institute - NRI, in Poznan, Poland (Spasibionek 2006). Two statistically important single nucleotide polymorphisms were detected in two FAD3 desaturase genes: (1) a $\mathrm{C}$ to $\mathrm{T}$ substitution in the third position of the sixth codon of the seventh exon in the BnaA. FAD3 gene and (2) a $\mathrm{G}$ to A transition in the 5' splice donor site of the sixth intron in the BnaC.FAD3 gene (Mikolajczyk et al. 2010). Allele-specific SNP markers were designed involving a two-step detection of the wild-type and mutant alleles in both loci by SNaPshot analysis comprising an independent and specific PCR amplifications of B. napus BnaA.FAD3 and BnaC.FAD3 gene fragments comprising the mutation sites, and detection of the mutant alleles analysis using locus-specific primers (Mikolajczyk et al. 2010). Strong negative correlation between the presence of mutated alleles in the $\mathrm{A}$ and $\mathrm{C}$ genomes and linolenic acid content was revealed by analysis of variance (Mikolajczyk et al. 2010).

In this paper we report detailed characteristics of the developed allele-specific markers by estimation of the additive and dominance effects of the FAD3 genes with respect to particular fatty acid content in seed oil. In addition, percentage of phenotypic variation of seed oil fatty acid composition accounted by particular allele-specific marker was calculated for wild-type and mutated alleles of BnaA. FAD3 and BnaC.FAD3, respectively.

\section{Materials and methods}

Plant material consisted of 450 winter oilseed rape plants: 36 canola-type $(00,7-10 \%$ of linolenic acid in seed oil) cultivars and 26 doubled haploid (DH) mutant low linolenic (LL, $\leq 3 \%$

Table 2 Additive (a) and dominance (d) effects of the BnaA.FAD3 (FAD3A) i BnaC.FAD3 (FAD3C) alleles on seed oil composition; particular fatty acid abbreviations (in the 'Trait' column) as in Table

\begin{tabular}{llllll}
\hline Trait & \multicolumn{2}{l}{ FAD3A } & & & \multicolumn{2}{l}{ FAD3C } \\
\cline { 2 - 3 } \cline { 5 - 6 } & $\mathrm{a}$ & & $\mathrm{d}$ & & $\mathrm{d}$ \\
\hline $\mathrm{C} 16: 0$ & $0.099^{\mathrm{a}}$ & $-0.251^{\mathrm{b}}$ & & 0.059 & $-0.255^{\mathrm{b}}$ \\
$\mathrm{C} 18: 0$ & $-0.157^{\mathrm{b}}$ & -0.002 & & $-0.121^{\mathrm{b}}$ & 0.025 \\
$\mathrm{C} 18: 1$ & -0.382 & $1.419^{\mathrm{b}}$ & & 0.436 & $1.551^{\mathrm{b}}$ \\
$\mathrm{C} 18: 2$ & $-1.136^{\mathrm{b}}$ & $-0.945^{\mathrm{a}}$ & & $-1.935^{\mathrm{b}}$ & $-1.117^{\mathrm{b}}$ \\
$\mathrm{C} 18: 3$ & $1.595^{\mathrm{b}}$ & -0.225 & & $1.571^{\mathrm{b}}$ & -0.180 \\
$\mathrm{C} 20: 1$ & -0.016 & 0.019 & & -0.013 & 0.007 \\
\hline
\end{tabular}

${ }^{\mathrm{a}}$ significant at 0.01 level; ${ }^{\mathrm{b}}$ significant at 0.001 level 
of linolenic acid) lines, as well as 26 DH lines developed from $F_{1}$ hybrid obtained as a result of crosses between 00 and LL lines in addition to 362 plants of $F_{2}$ and $\mathrm{F}_{3}$ segregating lines obtained from crosses of 00 and LL lines, from 2 to 10 plants of each line. Field experiments (2007/2008 and 2008/2009) were conducted in Karzniczka, Lagiewniki and Poznan, Poland (Mikolajczyk et al. 2010).

The fatty acid composition of mature seeds was determined by gas liquid chromatography of the methyl esters (Byczynska and Krzymanski 1969) using an Agillent Technologies $6890 \mathrm{~N}$ gas chromatograph (DB $2330 \mathrm{~m}$, ID $025,0.25 \mu \mathrm{m}$ layer capillary column) equipped with a Chemstation integrator.

Allelic forms of the BnaA.FAD3 and BnaC.FAD3 genes were identified by independent and specific PCR amplifications of the gene fragments comprising mutation sites and detection of the mutant alleles by SNaPshot analysis using locus-specific primers (Mikolajczyk et al. 2010). Regression analysis was done for the mutations in the FAD3 genes on fatty acid composition in seed oil. The analysis was performed both -1) individually, as well as 2) simultaneously for the two genes. The FAD 3 genes observations were treated as independent variables and considered in individual models (the first case), as well as in multiple regression model (the second case). The additive and dominance effects of the presence or absence of mutations in the FAD3 genes for particular fatty acid content in seed oil were estimated. The additive effect of each allel was estimated as half of the difference between homozygotes and we tested null hypothesis that the difference between mean values for homozygotes is equal to zero by two-sample $t$-test. The dominance effect was estimated as deviation of mean value for heterozygotes from grand mean. We tested a null hypothesis that mean value for heterozygotes is equal to grand mean by twosample $t$-test. The broad-sense heritability coefficients were calculated (Falconer and Mackay 1996). All calculations were carried out using GenStat v. 7.1 (Payne et al. 2003).

\section{Results and conclusions}

The presence of the mutated BnaA.FAD3 allele was associated with seed oil fatty acid composition with statistical significance, except for eicosenoic acid content $(\mathrm{C} 20: 1) \quad(\mathrm{P}=0.129)$ - possibly due to little content of eicosenoic acid in oilseed rape oil. At the same time, the mutant form of the BnaC.FAD3 gene was associated with stearic $(\mathrm{C} 18: 0)(\mathrm{P}<0.001)$, linoleic $(\mathrm{C} 18: 2)(\mathrm{P}<0.001)$ and linolenic acid $(\mathrm{C} 18: 3)(\mathrm{P}<0.001)$ content (Table 1). The percentage of variation in fatty acid composition accounted for the presence of mutations in the BnaA.FAD3 and BnaC. FAD3 genes by the SNaPshot analysis was considerable only for the linolenic acid content and equaled $35.6 \%$ and $39.3 \%$, respectively (Table 1 ), whereas the total percentage of variation in linolenic acid content was $53.2 \%$ when accounted for mutations in both genes simultaneously (Table 1). At the same time, the oleic acid content was markedly influenced by the presence of the mutated alleles of the FAD3 genes (Table 1) and it was not the case for the presence of the mutated BnaC.FAD3 alone (Table 1).

Additive effects of the mutated alleles of the BnaA. $F A D 3$ and BnaC.FAD3 genes were significant for stearic, linoleic and linolenic acid content and the signs of the effect values were the same for both genes (Table 2). Moreover, additive effect of the mutated allele of the BnaA.FAD3 gene was significant for the palmitic acid content (Table 2).

Dominance effects assessed for the presence of the mutated alleles were significant for palmitic, oleic and linoleic acid content (Table 2). The absolute values of the dominance effects were larger than the absolute values of the additive effects for palmitic and oleic acids content. However, it was not the case for stearic, linoleic and linolenic acids content.

Additive effects are fixed in the population as the number of homozygotes increases in successive generations (Bocianowski and Krajewski 2009) and insignificant dominance effects at early generations indicate high selectivity of the markers, which proved very useful for monitoring wild-type and mutant alleles of FAD3 desaturase. The markers will be further applied in oilseed rape breeding programs concerning diversification of seed oil fatty acid composition.

Open Access This article is distributed under the terms of the Creative Commons Attribution Noncommercial License which permits any noncommercial use, distribution, and reproduction in any medium, provided the original author(s) and source are credited.

\section{References}

Barret P, Delourme R, Brunel D, Jourdren C, Horvais R, Renard M (1999) Low linolenic acid level in rapeseed can be easily assesed through the detection of two single base substitution in FAD3 genes. Proc. 10th International Rapeseed Congress, Canberra, Australia, 26-29.09.1999. [CD-ROM]. Available at www.regional. org.au/au/gcirc/4/385.htm

Bocianowski J, Krajewski P (2009) Comparison of the genetic additive effect estimators based on phenotypic observations and on molecular marker data. Euphytica 165:113-122 
Byczynska B, Krzymanski J (1969) A fast method for obtaining of fatty acids methyl esters to be analysed by gas chromatography method (in Polish). Tluszcze Jadalne XIII:108-114

Falconer DS, Mackay TFC (1996) Introduction to quantitative genetics. Addison-Wesley, Essex, pp 26-57

Hu X, Sullivan ML, Gupta M, Thompson SA (2006) Mapping of the loci controlling oleic and linolenic acid contents and development of fad 2 and fad 3 allele-specific markers in canola (Brassica napus L.). Theor Appl Genet 113:497-507

Jourdren C, Barret P, Horvais R, Delourme R, Renard M (1996) Identification of RAPD markers linked to linolenic acid genes in rapeseed. Euphytica 90:351-357
Mikolajczyk K, Dabert M, Karlowski WM, Spasibionek S, Nowakowska J, Cegielska-Taras T, Bartkowiak-Broda I (2010) Allele-specific SNP markers for the low linolenic mutant genotype of winter oilseed rape. Plant Breeding 129:502-507

Østergaard L, King GJ (2008) Standarized gene nomenclature for the Brassica genus. Plant Methods 4:10

Payne R, Murrey D, Harding S, Baird D, Soutou D, Lane P (2003) GenStat for Windows (7th edition) - Introduction. VSN International, Oxford, England

Spasibionek S (2006) New mutants of winter rapeseed (Brassica napus L.) with changed fatty acid composition. Plant Breeding 125:259-267 